\title{
UAV Conflict Detection and Resolution Based on Geometric Approach
}

\author{
Jung-Woo Park*, Hyon-Dong $\mathrm{Oh}^{* *}$ and Min-Jea Tahk*** \\ Department of aerospace Engineering, \\ Korea Advanced Institute of Science and Technology, \\ Daejeon, Korea, 305-701
}

\begin{abstract}
A method of conflict detection and resolution is described by using simple geometric approach. Two UAVs are dealt with and considered as point masses with constant velocity. This paper discusses en route aircraft which are assumed to be linked by real time data bases like ADS-B. With this data base, all UAVs share the information each other.

Calculating PCA (Point of Closest Approach), we can evaluate the worst conflict condition between two UAVs. This paper proposes one resolution maneuvering logic, which can be called 'Vector Sharing Resolution'. In case of conflict, using miss distance vector in PCA, we can decide the directions for two UAVs to share the conflict region. With these directions, UAVs are going to maneuver cooperatively. First of all, this paper describes some '2-D' conflict scenarios and then extends to '3-D' conflict scenarios.
\end{abstract}

Key Word : Vector Sharing Resolution, Cooperative Collision Avoidance, Point of Closest Approach (PCA), Sphere Protected Zone

\section{Introduction}

There are many researches for 'Free Flight' [1]. Many methodologies and skills are published up to the present. For example, there are many studies accomplished that finding optimal trajectories by using many optimal theories, probabilistic modeling, applying potential field, and so on.

'Conflict' can be defined as a "predicted violation of a separation assurance standard" [2]. So if the protected zone is violated, each UAV should solve the violation using proper way to avoid the conflict.

For the Free Flight, it is very essential task to understand geometric relations between two UAVs in a conflict. In this paper, using PCA method[3], we calculate the miss distance vector of two UAVs and the time to take. If the magnitude of miss distance vector is smaller than the minimum separation which should be guaranteed, it is considered as a conflict that can bring about collision between UAVs.

Therefore this paper discusses a method to resolve the conflict, essentially to avoid the collision between a pair of UAVs by using simple geometric sense.

* Student in the Ph. D Course

** Student in the Master's Course

*** Professor

Email : mjtahk@fdcl.kaist.ac.kr

Tel : +82-42-350-3718 FAX : $+82-42-350-3710$ 


\section{System Modeling}

In this paper, two UAVs with constant velocities toward their goal positions are considered in a conflict condition when they are within a protected zone. Generally, aircraft's 'Protected Zone' is currently sized by $5 \mathrm{nmi}$ (about $9.26 \mathrm{~km}$ ) horizontally and $2000 \mathrm{ft}$ (about $0.61 \mathrm{~km}$ ) vertically [4], but for the simplistic, it is taken to be a sphere of specified radius about $5 \mathrm{nmi}$ in this paper. Initially the positions and velocities are assumed to be informed by certain broadcasting systems like ADS-B, and the information from such as GPS is assumed to be quite exact.

The point-mass equations of motion are derived with respect to a coordinate system shown in Fig. 1. The point-mass UAV equations are:

$$
\begin{aligned}
V & =\sqrt{V_{H}^{2}+V_{V}^{2}} \\
\dot{x}=V_{H} \cos \gamma, \dot{y} & =V_{H} \sin \gamma, \dot{z}=V \sin \theta=V_{V} \\
\dot{\gamma} & =\frac{g \tan \phi}{V_{H}}
\end{aligned}
$$

where $\theta$ is UAV's pitch angle, $\gamma$ is heading angle, and $\phi$ is bank angle. To generate heading angle change, bank command is given as an input, and pitch command is given to generate pitch angle. The detail equations are:

$$
\begin{aligned}
& \dot{\phi}=\frac{1}{N}\left(\phi_{\text {com }}-\phi\right) \\
& \dot{\theta}=\frac{1}{M}\left(\theta_{\text {com }}-\theta\right)
\end{aligned}
$$

The equations consider the delay of actual UAV's dynamics. It is shown that time constant is assumed to be $\mathrm{N}$ and $\mathrm{M}$ seconds for both dynamics at Eq. (4) and Eq. (5).

The relative distance is simply given as:

$$
R_{r e l}=\sqrt{\left(x_{A}-x_{B}\right)^{2}+\left(y_{A}-y_{B}\right)+\left(z_{A}-z_{B}\right)^{2}}
$$

With this relative distance, we can judge whether it is in a conflict condition or not.

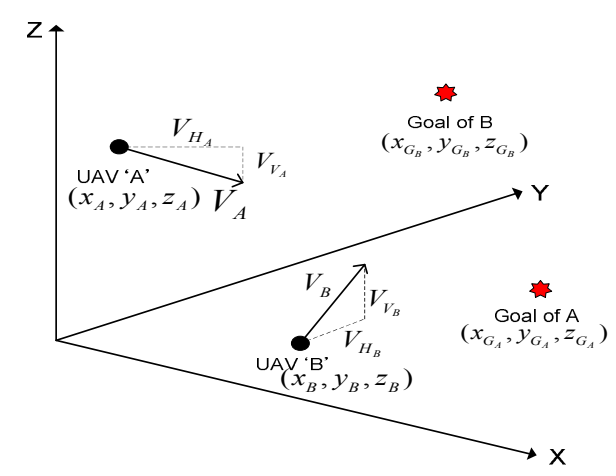

Fig. 1. Geometric View of Basic Conception of Two UAVs

\section{Collision Detection, Resolution, and Guidance to the way-point}

It is assumed that two UAVs are in an encounter with each other, and they are heading to their velocity direction which means there's no sideslip for UAVs. The geometry is illustrated in Fig. 2. 


\section{Collision Detection}

When two UAVs are getting closer, if we can calculate the minimum distance passed by each other, it can be judged whether collision can be occurred or not. For this, we can get the miss distance from PCA(Point of Closest Approach).

The miss distance vector $\vec{r}_{m}$ is defined:

$$
\vec{r}_{m}=\hat{c} \times(\hat{r} \times \hat{c})
$$

where $\vec{r}$ is the relative distance vector and $\hat{c}$ is the unit vector in the direction of the relative velocity vector $\vec{c}$ from $\mathrm{UAV}^{\prime} \mathrm{A}^{\prime}$ to $\mathrm{UAV}$ ' $\mathrm{B}^{\prime}$. Additionally, we can know easily that the miss vector $\vec{r}_{m}$ and the relative velocity vector $\vec{c}$ is orthogonal:

$$
\vec{r}_{m} \cdot \vec{c}=0
$$

With the relation between $\vec{r}_{m}$ and $\vec{r}$, we can calculate the time to closest approach $\tau$ :

$$
\vec{r}_{m}=\vec{r}+\vec{c} \cdot \tau
$$

With Eq.(8) and (9), finally we get:

$$
\tau=-\frac{\vec{r} \cdot \vec{c}}{\vec{c} \cdot \vec{c}}
$$

At the Eq. (10), when two of UAVs are getting closer, $\tau>0$, and when two of UAVs are getting further, $\tau<0$. Therefore, when $\tau>0$, we have to check whether there's a chance to have an event of conflict or not. When $\tau<0$, we can guess that there's no risk to have an event of collision at all.

If the magnitude of $\vec{r}_{m}$ is less than specified minimum separation distance $r_{\text {safe }}$, two UAVs are considered in a conflict condition.

- Conflict condition:

$$
r_{\text {res }}=r_{\text {safe }}-\left\|\vec{r}_{m}\right\|>0 \text { and } \tau>0
$$

where $r_{\text {res }}$ is the rest distance after subtraction of $\left\|\vec{r}_{m}\right\|$ from $r_{\text {safe }}, r_{\text {res }}$ will be called "Unresolved Region" in this paper.

\section{Collision Resolution}

If $r_{\text {res }}>0$, conflict resolution maneuvering should be accomplished. In Fig. 2, we can intuitively know the direction where each of UAVs has to go. UAV 'A' may turn to the left to avoid collision and UAV 'B' may turn to the left too. It can be easily figured out if we think about the motion of UAVs with respect to the miss distance vector.

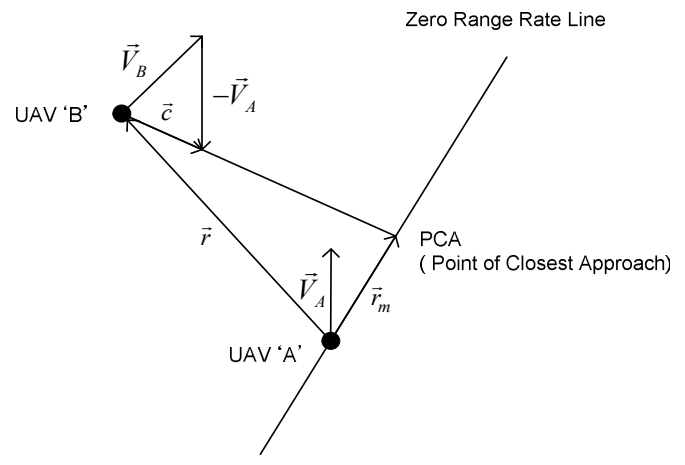

Fig. 2. Relative motion of two UAVs 
To larger the miss distance, UAV 'A' and 'B' have no choice except being headed for left. If UAV 'A' makes a turn to the right, it takes a very long distance roundabout way or it collides with UAV 'B'. The case of UAV ' $\mathrm{B}$ ' is also same logic.

To solve this problem, it is proposed that the conflict resolution maneuvering lies on the line of miss distance vector. This will be called as "Vector Sharing Resolution" in this paper and it is described in Fig. 3.

In the Fig. 3, to share the unresolved region, two UAVs should at least head for the direction of unit vector $\vec{U}_{A}$ and $\vec{U}_{B}$. This indicates that this logic gives each UAV a direction to solve the conflict condition.

Vector sharing resolution is achieved as defining the vector $\vec{r}_{V S A}$ and $\vec{r}_{V S B}$ in Fig. 3:

- For UAV 'A'

$$
\vec{r}_{V S A}=\frac{\left|\vec{V}_{B}\right|}{\left|\vec{V}_{A}\right|+\left|\vec{V}_{B}\right|} \frac{r_{r e s}}{\left|\vec{r}_{m}\right|}\left(-\vec{r}_{m}\right)
$$

- For UAV 'B'

$$
\vec{r}_{V S B}=\frac{\left|\vec{V}_{A}\right|}{\left|\vec{V}_{A}\right|+\left|\vec{V}_{B}\right|} \frac{r_{r e s}}{\left|\vec{r}_{m}\right|}\left(-\vec{r}_{m}\right)
$$

- A relation between and :

$$
r_{\text {safe }}=\left|\vec{r}_{V S A}\right|+\left|\vec{r}_{V S B}\right|+\left|\vec{r}_{m}\right|
$$

By the means of that the slower UAV takes the more sharing, the sharing is done. It is because slower UAV can do avoidance maneuver more than faster one with same time.

Finally we get the unit vector $\vec{U}_{A}$ and $\vec{U}_{B}$ :

- For UAV 'A'

$$
\vec{U}_{A}=\frac{\vec{V}_{A} \cdot \tau+\vec{r}_{V S A}}{\left|\vec{V}_{A} \cdot \tau+\vec{r}_{V S A}\right|}
$$

- For UAV 'B'

$$
\vec{U}_{B}=\frac{\vec{V}_{B} \cdot \tau+\vec{r}_{V S B}}{\left|\vec{V}_{B} \cdot \tau+\vec{r}_{V S B}\right|}
$$

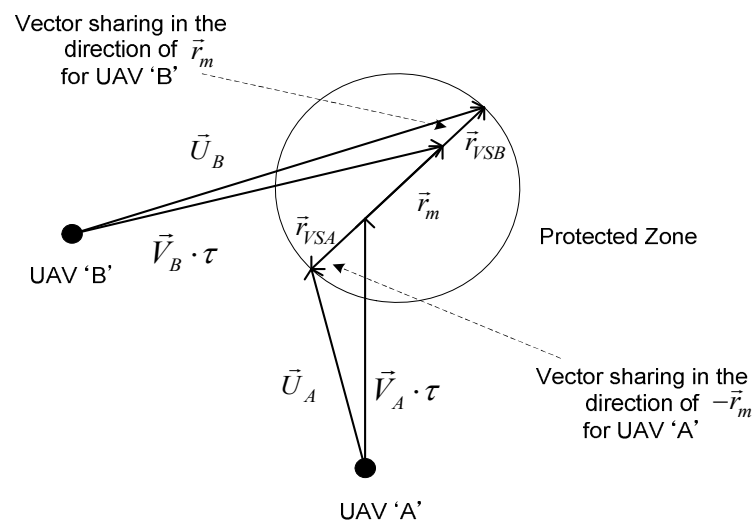

Fig. 3. Resolution Maneuvering 
If two UAVs are going to have a direct head-on collision $\left(\vec{r}_{m}=0\right.$ ), by disturbance making process we make two UAVs have non-zero miss distance vector. Detail is as follows:

$$
\vec{U}=\frac{\vec{V}+0.01 \times(\vec{h} \times \vec{V})}{|\vec{V}+0.01 \times(\vec{h} \times \vec{V})|}
$$

where $\vec{h}$ is the unit vector of $z$-direction. In real flight, by certain maneuver of one of UAVs, this problem can be resolved. Additionally, if it is doubt to the chattering or not complete information from $\mathrm{ADS}-\mathrm{B}$, we define certain region to be dealt with as zero miss distance region.

\section{Guidance to the way-point}

In the case that the conflict probability is zero or the time to PCA is negative value, own aircraft is guided to the way-point. The acceleration command of PNG is given as :

$$
\vec{a}_{g}=N \overrightarrow{\vec{\sigma}}_{G} \times \vec{V}
$$

\section{Verification for Optimal Maneuver to Resolve}

We can consider the optimal problem to maximize the miss distance at the end of resolution maneuver.

$$
\min _{a} J=-\frac{1}{2}\left|\vec{r}_{m}\right|_{f}^{2}
$$

where ' $\mathrm{a}$ ' is acceleration vector as input with constant magnitude of ' $\mathrm{A}$ '. Then we can derive the equation of Hamiltonian.

$$
H=-\vec{r}_{f} \cdot \vec{v}-A\left(t_{f}-t\right) \vec{r}_{f} \cdot \vec{e}
$$

where $\vec{e}$ is the unit vector of acceleration vector. Therefore we conclude that acceleration along the miss distance vector can minimize the Hamiltonian so that it can be the optimal solution for maximum miss distance.[5]

This is not sudden result. As described before, it is verified that the most efficient way is to avoid each other along the miss distance vector since the miss distance vector is perpendicular to the relative velocity vector. Therefore the strategy of the algorithm which is introduced on 'Vector Sharing Resolution' is acceptable.

\section{Application to the UAV Dynamics}

It is informed of the directions of now-going and modified. Now-going one is the direction of velocity $\vec{V}$ and modified one is the direction of $\vec{U}$.

\section{1) Horizontal Maneuver}

With these two vectors we can calculate the LOSH (Line Of Sight on Horizontal) angle between $\vec{V}$ and $\vec{U}$. For each UAV, the LOSH is defined:

$$
\lambda=\operatorname{signum}\left(\left(\vec{V}_{H} \times \vec{U}_{H}\right)_{z}\right) \arccos \left(\frac{\vec{V}_{H} \cdot \vec{U}_{H}}{\left|\vec{V}_{H}\right|}\right)
$$

where subscript ' $\mathrm{H}$ ' means horizontal element.

By the LOSH angle found we decide the bank command as an input. It is assumed that each UAV has its maximum bank angle of 45 degree. Therefore with the maximum bank angle, we can calculate the maximum heading angle change for 1 second: 
Table 1. Horizontal Maneuver Options

\begin{tabular}{|c|c|}
\hline Range of LOSH Angle & Bank Command \\
\hline \hline$\lambda<-\gamma_{\max }$ & $\phi_{\text {com }}=-45^{\circ}$ \\
$-\gamma_{\max } \leqq \lambda \leqq \gamma_{\max }$ & $\phi_{\text {com }}=\frac{\lambda}{\gamma_{\max }} \times 45^{\circ}$ \\
\hline$\gamma_{\max }<\lambda$ & $\phi_{\text {com }}=45^{\circ}$ \\
\hline
\end{tabular}

$$
\gamma_{\max }=\frac{g}{\left|\vec{V}_{H}\right|} \text {, for } 1 \text { second and } \phi_{\max }
$$

where $\mathrm{g}$ is the gravity acceleration and $\vec{V}_{H}$ is the horizontal velocity.

Keeping on this result, the horizontal maneuver logic is followed as:

An UAV can change the bank angle easily, so we set the time constant $\mathrm{N}$ of 1 second in Eq. (4).

\section{2) Vertical maneuver}

With unit vector $\vec{U}$ for each UAV, we can obtain the pitch angle(LOSV similar to LOSH) required. Required pitch angle can be expressed as:

$$
\text { LOSV angle }=\theta_{\text {req }}=\arctan \left(\frac{\vec{U}_{V}}{\left|\vec{U}_{H}\right|}\right)
$$

Vertical motion is hard to change fast. So we have to deal with the change of pitch angle carefully. At the vertical maneuver, we set the time constant of $\mathrm{M}$ in Eq. (5) by the required pitch angle. Vertical maneuver logic is followed as:

Table 2. Vertical Maneuver Options

\begin{tabular}{|c|c|}
\hline Required Pitch Angle & Time constant, $M$ \\
\hline \hline $0^{\circ} \leqq\left|\theta_{\text {req }}\right|<15^{\circ}$ & 1 second \\
\hline $15^{\circ} \leqq\left|\theta_{r e q}\right|<30^{\circ}$ & 2 second \\
\hline $30^{\circ} \leqq\left|\theta_{r e q}\right|<45^{\circ}$ & 3 second \\
\hline $45^{\circ} \leqq\left|\theta_{\text {req }}\right|$ & 4 second \\
\hline
\end{tabular}

As pitch angle changes, the horizontal velocity and the vertical velocity also change. The velocity element change is described as:

$$
\left|\vec{V}_{V}\right|=|\vec{V}| \sin \theta \text { and }\left|\vec{V}_{H}\right|=|\vec{V}| \cos \theta
$$

\section{Numerical Results}

The conflict detection and resolution algorithm discussed in previous sections are evaluated in two sample encounter scenario in this section. Simulation has been accomplished with initial information about position and velocity for both UAVs. Two UAVs are assumed to head toward their goal position. And the integrated positions and velocities have been treated as broadcasted information. Minimum separation distance is $5 \mathrm{nmi}$ (about $9.26 \mathrm{~km}$ ) as defined. It is assumed that UAV detects conflict situation where time to PCA is less than $150 \mathrm{sec}$. Also it is assumed that the interval for updating information from ADS-B is $2 \mathrm{sec}$ and the command for maneuver is generated right after the updating. Between the intervals, UAVs maneuver with the commands for bank and pitch during $2 \mathrm{sec}$. It is for the consistent maneuvering not to change the bank and pitch angle suddenly during the conflict resolution. Additionally, if not in conflict but existence of 
conflict possibility (where time to PCA is positive), the command for resolution is sustained. All simulations for $2-\mathrm{D}$ and $3-\mathrm{D}$ are accomplished assuming all scenarios are non-cooperative cases since it is easier to avoid each other cooperatively so that the non-cooperative maneuver guarantees the successful conflict resolution of the cooperative cases.

\section{Scenario \#1 : 2-D Conflict}

Table 3. Problem Definition for 2-D

\begin{tabular}{c|ccc}
\hline \multirow{3}{*}{ UAV 'A' } & Initial Position & $(0,0,0)$ & $\mathrm{km}$ \\
& Initial Velocity & $(150,150,0)$ & $\mathrm{m} / \mathrm{s}$ \\
& Goal position & $(75,75 ., 0)$ & $\mathrm{km}$ \\
\hline \hline & Initial Position & $(0,100,0)$ & $\mathrm{km}$ \\
UAV 'B' & Initial Velocity & $(150,-145,0)$ & $\mathrm{m} / \mathrm{s}$ \\
& Goal position & $(75,27.5,0)$ & $\mathrm{km}$ \\
\hline
\end{tabular}

- Results:

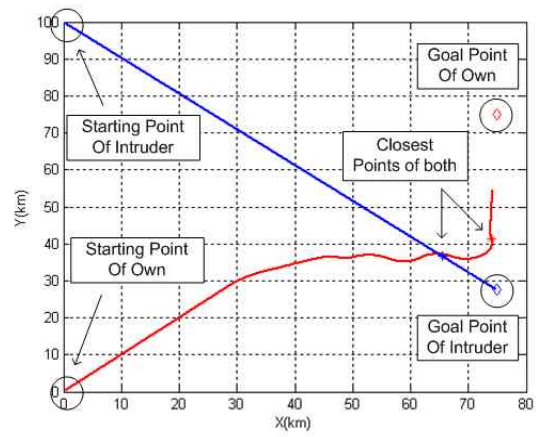

Fig. 4. Trajectories of both UAVs

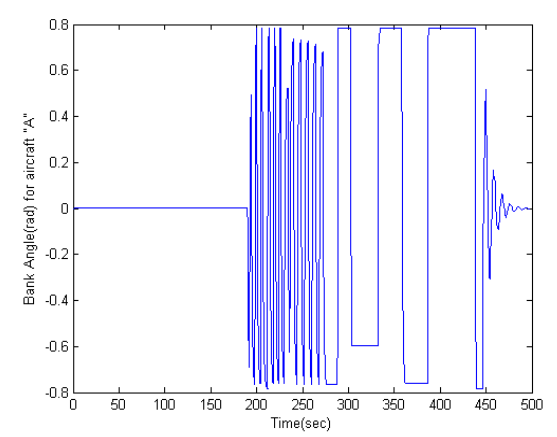

Fig. 6. Bank Angle profile for Own UAV

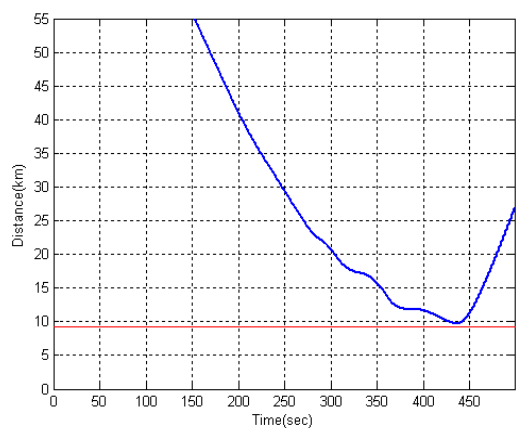

Fig. 5. Relative Distance

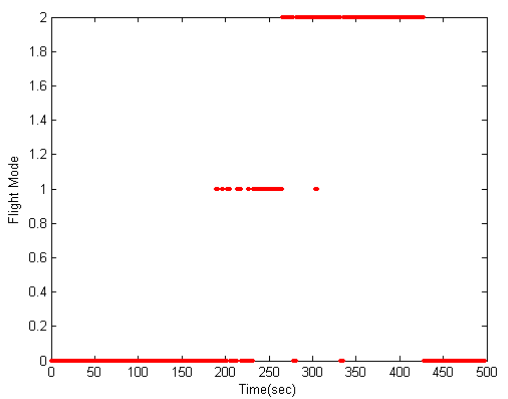

Fig. 7. Flight Mode

Own UAV turns to the right to avoid the conflict. The trajectory obtained is expected pattern and the relative distance also never invades the minimum separation region. Flight mode in Fig. 7 demonstrates that, if UAV is homing to the way point, it is indicated as the value of 0 , if on the stage of conflict resolution with the algorithm which is introduced in this paper, flight mode is expressed as the value of 1 , and if not in conflict but existence of conflict possibility (where time to PCA is positive), the value of 2. From this result, it is known that own UAV doesn't detect the conflict for a while. And then, it is detected to be in conflict and avoidance maneuver is performed. It is shown also in the bank profile for own UAV. When the avoidance algorithm is applied (in the case of conflict detected), own UAV starts bank maneuver. If there's no risk of conflict after resolution for a while, the guidance logic is applied to the way point where time to PCA is negative. 
It is repeated during some period. This maneuver is reflected to the sudden bank maneuver during the time from $200 \mathrm{sec}$ to $270 \mathrm{sec}$. And then bank angle is sustained under the condition where not in conflict but time to PCA is positive. Finally after own UAV goes through the risk of conflict, it is guided to the way point by PNG algorithm (after about $420 \mathrm{sec}$ from start).

\section{Scenario \#2 : 3-D Conflict}

Table 4. Problem Definition for '3-D'

\begin{tabular}{cccc}
\hline & Initial Position & $(0,0,10)$ & $\mathrm{km}$ \\
UAV 'A' & Initial Velocity & $(150,100,10)$ & $\mathrm{m} / \mathrm{s}$ \\
& Goal position & $(75,5015)$ & $\mathrm{km}$ \\
\hline \hline \multirow{3}{*}{ UAV 'B' } & Initial Position & $(30,0,10)$ & $\mathrm{km}$ \\
& Initial Velocity & $(-150,90,10)$ & $\mathrm{m} / \mathrm{s}$ \\
& Goal position & $(-45,45,15)$ & $\mathrm{km}$ \\
\hline
\end{tabular}

- Results:

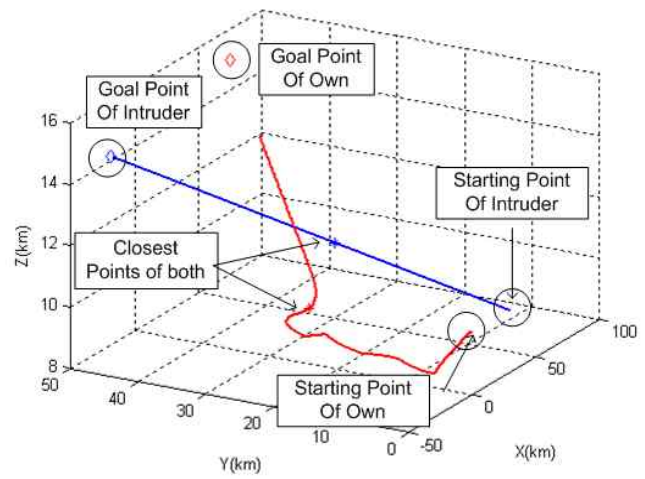

Fig. 8. Trajectories of both UAVs

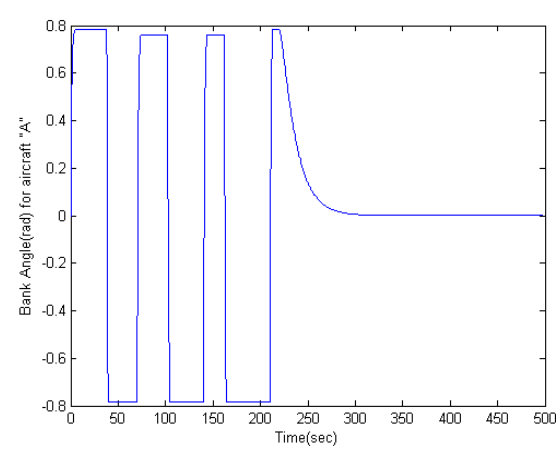

Fig. 10. Bank Angle Profile for Own UAV

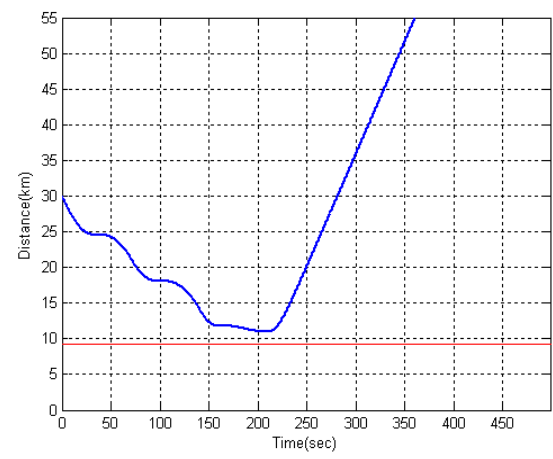

Fig. 9. Relative Distance

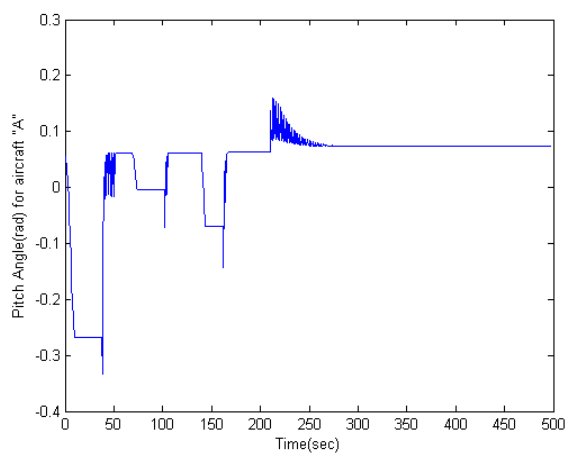

Fig. 11. Pitch Angle Profile for Own UAV

From the start simulation, conflict is detected and own UAV starts resolution maneuver. After a little pitch and bank maneuver conflict is resolved but there is still risk of conflict (time to PCA is positive). Therefore the bank and theta angle is sustained for a while. After the risk of conflict is eliminated totally, the guidance logic is dominant. 


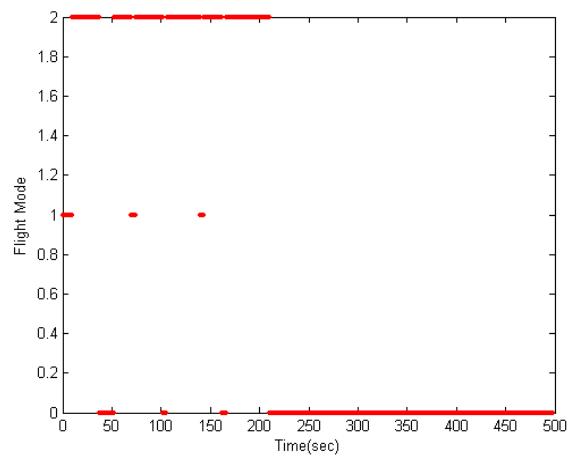

Fig. 12. Flight Mode

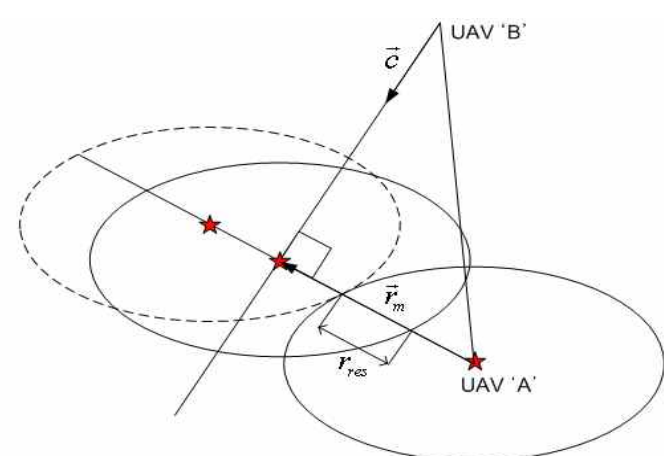

Fig. 13. Ellipsoidal Resolution in 2D

\section{Acknowledgement}

This research(paper) was performed for the Smart UAV Development program, one of the 21st Century Frontier R\&D Programs funded by the Ministry of Knowledge Economy.

\section{Conclusions}

Collision avoidance is performed by accomplishing the conflict resolution algorithm described in this study. Keeping and not losing the minimum separation distance, we settle up the conflict between UAVs. There are many developed methodologies and skills to avoid the conflict. The geometric approach is also one of the ways. In this paper, using geometric method, the conflict resolution maneuver is accomplished successfully. Using equations for PCA, the algorithm for conflict detection and resolution is induced by intuition simply. It means that it is possible and effective to resolve the conflict between UAVs successfully. But there are still tasks to solve furthermore.

Since this paper deals with simple dynamics for the UAVs, more realistic and well approached dynamics should be tested, especially in the pitch dynamics. And the other form of protected zone such as ellipsoidal can be considered. Fig. 13 shows the concept of resolution with ellipsoidal protected zone. It is simply verified that we can apply the 'Vector Sharing Resolution' algorithm with ellipsoidal protected zone easily sustaining the main ideas for resolution.

Finally 'Vector Sharing Resolution' is one of many resolution algorithms for collision avoidance, and it gives one possibility to resolve the conflict.

\section{References}

1. Lee C. Yang and James K. Kuchar, "Prototype Conflict Alerting System for Free Flight", American Institute of Aeronautics and Astronautics, Inc., 1997.

2. Wallace E. Kelly III, Rockwell Collins, Cedar Rapids, and Iowa, "Conflict Detection and Alerting for Separation Assurance Systems”, Digital Avionics Systems Conference, St. Louis, 1999.

3. Jimmy Krozel and Mark Peters, "Strategic Conflict Detection and Resolution for Free Flight", Seagull technology, Inc., 1997.

4. Douglas R. Isaacson and Heinz Erzberger, "Design of A Conflict Detection Algorithm for The Center/Tracon Automation System”, NASA Ames Research Center, 1997.

5. A.W.MERZ, "Maximum-Miss Aircraft Collision Avoidance", Dynamics and Control, Vol. 1, pp. 25-34, 1991. 